\title{
Distinguish of Fingerprint Based on Artificial Neural Networks
}

\author{
Zahraa Mazen Al-Qattan
}

College of Computer Sciences and Mathematics

University of Mosul, Mosul, Iraq

Received on: 14/04/2013

Accepted on: 24/06/2013

\begin{abstract}
In this research use one of artificial intelligence techniques which is artificial neural networks was used to distinguish fingerprint from a range of fingerprints belong to a unified database, based on a set of properties to the texture of image and which are extracted and analyzed using co-occurrence matrix (Event), These properties are (contrast ,correlation, determined, homogeneity), and after extracting properties, a combination of neural networks (Cascade Neural Network CNN and Radial Basis Functions netwoek RBFN and Elman Neural Network ENN) used to distinguish fingerprint, and the results of training $100 \%$ for the three networks after being trained on the network (18) sample where each person(3)samples.

Network efficiency was measured in recognition by using scale (training rate) and scale (recognition rate RR) for comparison between these networks to see the best network in the recognition.
\end{abstract}

Keywords: Fingerprint, Artificial Neural Networks.

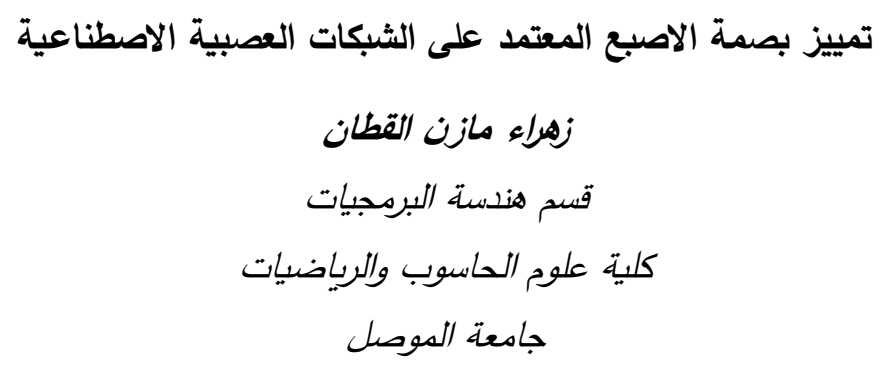

201316124 : تاريخ قبول البحث

$2013 \backslash 4 \backslash 14$ : تاريخ استلام البحث

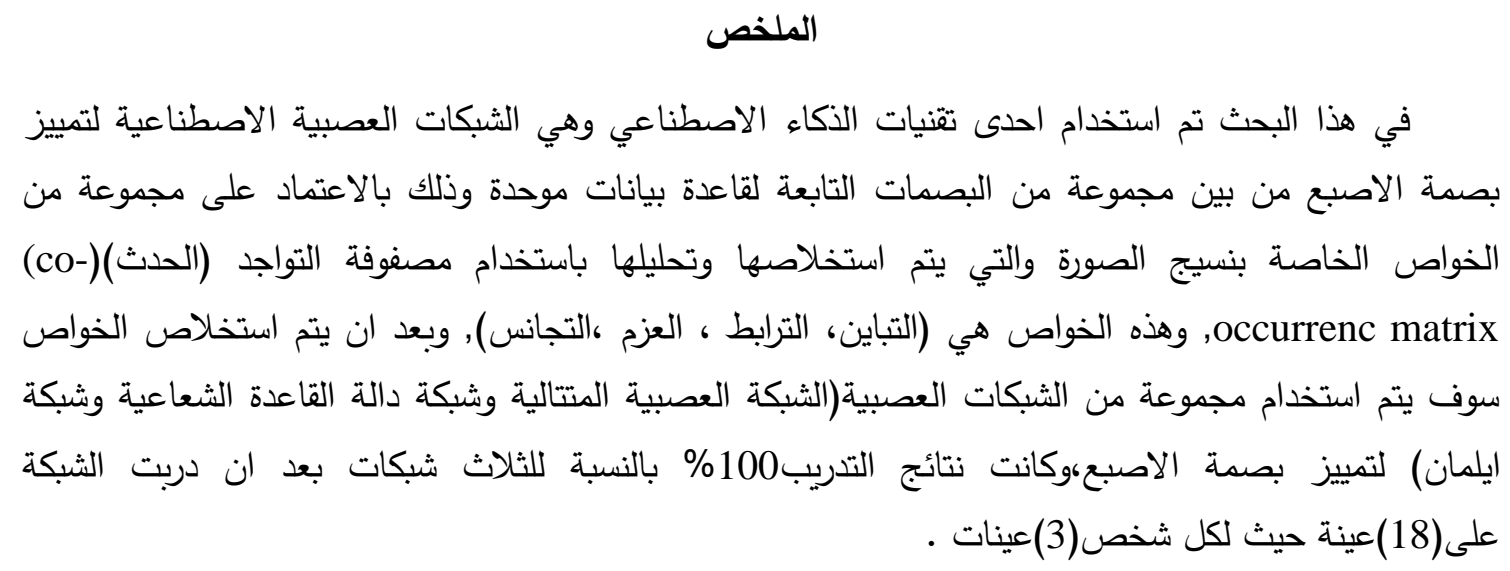


تم قياس كفـاءة الثـبكة في التمييز باستخدام مقيـاس نسبة التـدريب (training التمييز (recognition rate RR) للمقارنة بين هذه الثبكات لمعرفة افضل شبكة في التمييز • باسئ الكلمات المفتاحية: بصمة الاصبع، شبكة عصبية اصطناعية.

1. المقدمة

ان تمييز الانماط هو احد فروع علم معالجة الصور والذكاء الاصطناعي حيث يهدف الى ايجاد او تطوير تقنيات التعرف على نمط او شكل معين [1]. وان من التطبيقات المهمة والمفيدة في تمييز الانماط هي تمييز الحروف والتعرف على الاشكال والاشخاص وكذلك يستخدم في المجالات الطبية.

لقد عرف ألإنسان بصمات الأصـابع والكف وباطن القدم منذ عصر ما قبل التاريخ ، وقد أكد الباحثون

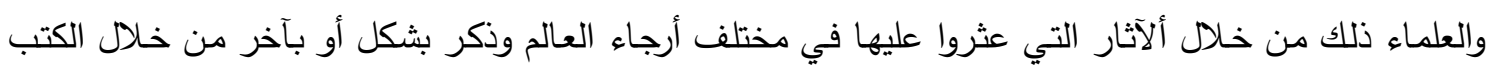

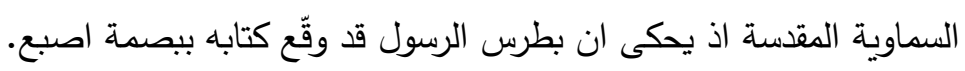
وتعد بصمات الاصابع والكف وباطن القدم ذات اهمية كبيرة في تحقيق شخصية الفرد اذ ثبت علميا وعمليا انه ليس هناك شخصان لهما طبعتان منطابقتان لاصبعين في الخطوط والمميزات حتى لو كان الثخصـان توأمين ،

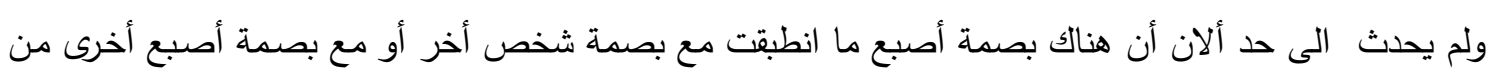
أصابع نفس الثخص. ونتيجة لتطور المجتمع فقد تطورت معه استعمال بصمات ألأصابع مثل العلوم الأخرى ويسمى علم بصمة

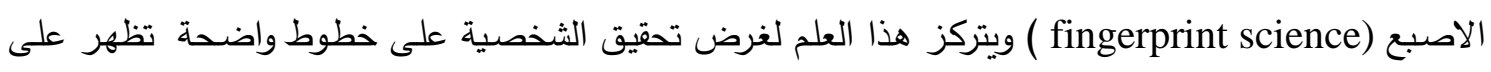

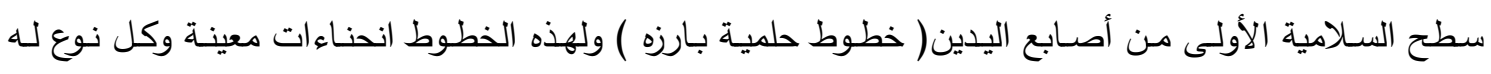
خصائص مميزه من حيث الثكل][1].

ان الهدف من البحث هو تمييز بصمة الاصبع باستخدام الشبكات العصبية الاصطناعية وبالاعتماد على هئ هني

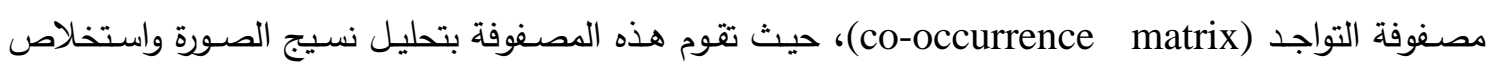
خواصها ،ومن ثم اعتمـاد هذه الخواص كمدخلات الى الشبكات العصبية المستخدمة في البحث لغرض عملية التمييز

\section{2. - 2. 20 2 الاعمال السابقة}

قدم الباحثون . Sushmita M., Sanker k. and Malay k طريقة لتصنيف بصمة الاصبع باستخدام

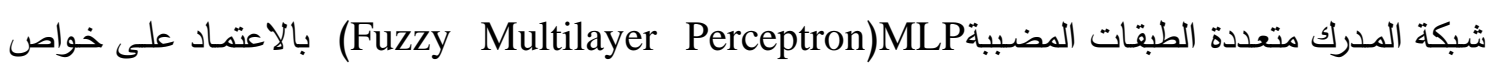

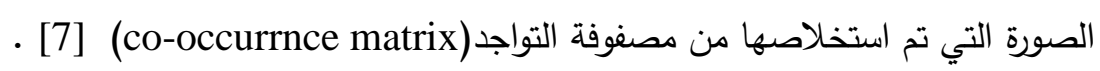
قام الباحثان Mehran Y. and Kazem G باستخدام مصفوفة التواجد في تصنيف بصمة الاصبع .حيث اعتمد على قيم الخواص المستخلصة من هذه الثبكة في تعليم الثبكة العصبية المستخدمة في تصنيف بصمة لتصني

الاصبع حسب انواعها المختلفة [8].

اما الباحثون Fakheri , Mashoufi B., Sedgi T. فقد قدموا من خلال بحثهم الموسوم تطبيق جديد لتمييز بصمة الاصبع(ANovel Approach for Fingerprinting Recognition) تمييز لبصمة الاصبع 
باستخدام شبكة الانتشار العكسي (Back-probagation algorithim) بعد ان يتم استخلاص الخواص من

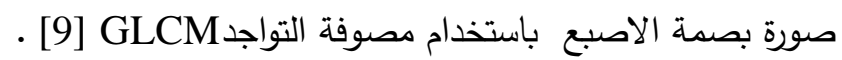

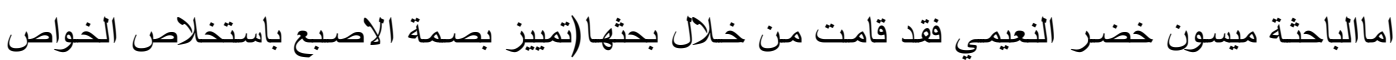
وباعتماد الثبكات العصبية) اولا بعملية ازالة الضوضاء من الصورة باستخدام الفلترالمتوسط ومن ثم التحيف لصورة

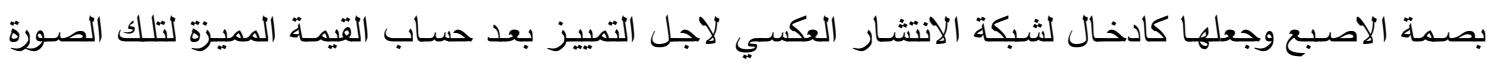
كطريقة لاستخلاص الخواص [2]. 3. 3. الثكل العام لبصمة الاصبع يلاحظ الثكل العام لبصمة الإصبع إن سطحه مكسو بخطوط دقيقة متوازيـة ترتفع عن سطح جلد البشرة

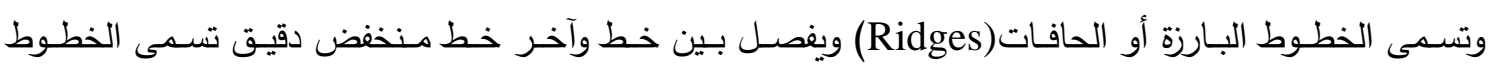
المنخفضة أو الثقوق (Furrows) او الوديان (Valleys) ولا تسير في مجراها باستقامة واحدة إنما تكوّن أشكالا

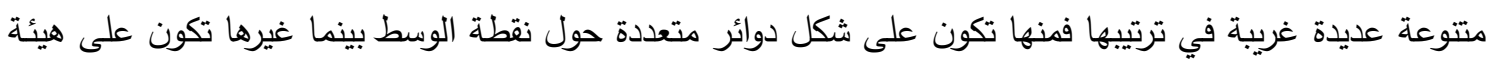

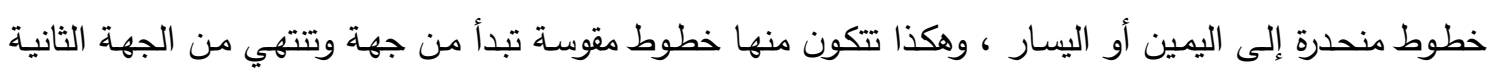
[1] [ . وتقسم بصمة الاصبع الى عدة انواع هي:

1- الدقوسات (Arches) والتي تضم : المقوسات البسيطة (Plain arch) ، المقوسات المرتفعة (الخيمة) (Tented arch)

2- المنحدات( Loops) والتي تضم : المنحدرات الكعبرية ( Radial Loops)و المنحدرات الزندية (Lunar) .Loops 3- المستديرات( Whorl).والثكل الاتي يوضح بعض الاثكال المختلفة لبصمة الاصبع [1].

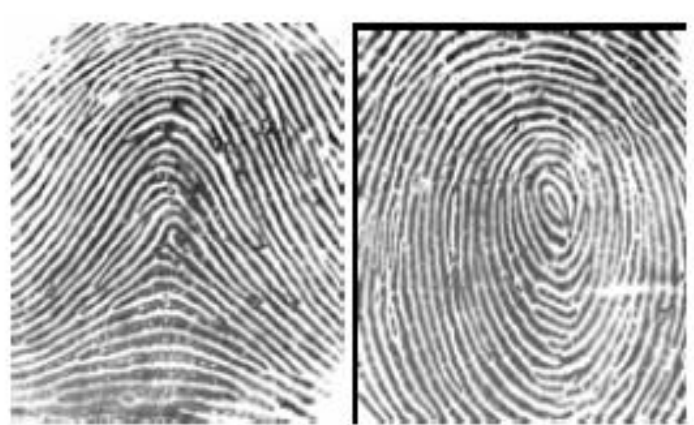

Arch

Whorl

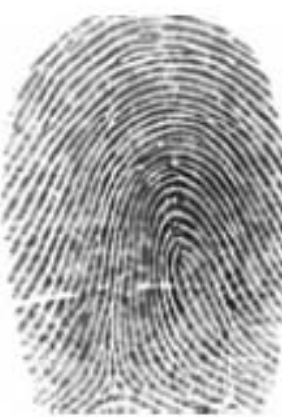

Right Loop

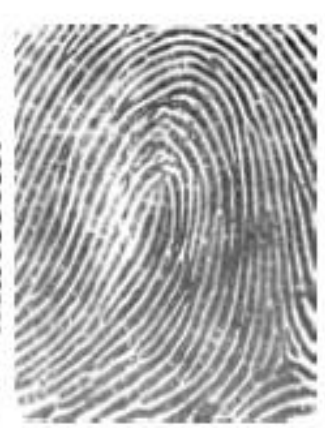

Left Loop

الثكل (1) الاثكال المختلفة لبصمة الاصبع

4. الخصائص الثخصية لبصمات الأصابع والمعايير الحيوية

هناك عدة مميزات لنمط بصمة الاصبع تجعلها اكثر استخداما من غيرها من المعايير الحيوية الاخرى مثل الوجه والاذن والصوت وغيرها لاغراض الامن والتحقيق وهي : 


\section{• • (Unexchangeability ( عدم التغير (l)

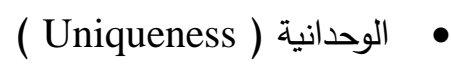 \\ وهناك أنواع مختلفة من المعايير الحيوية نذكر أههها:}

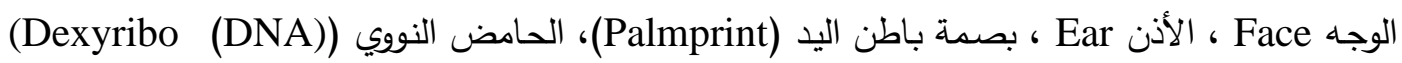

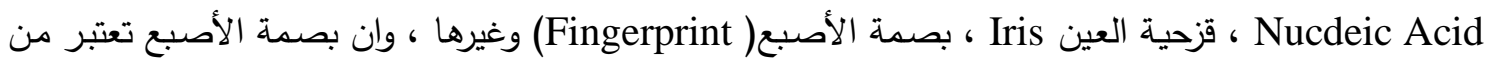

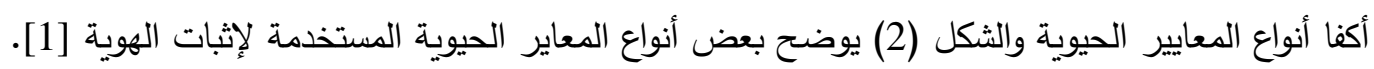
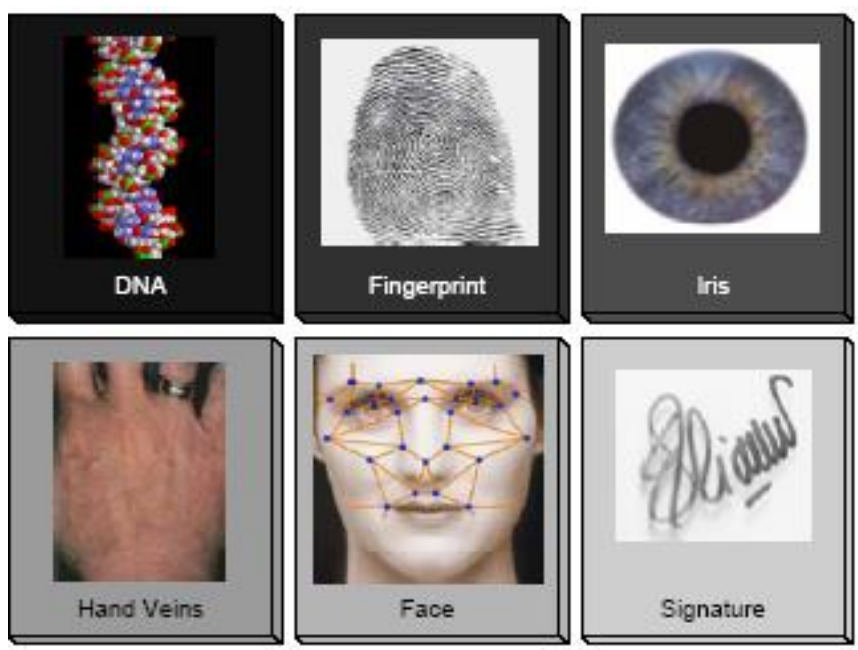

أنواع مختلفة من المعاير الحيوية
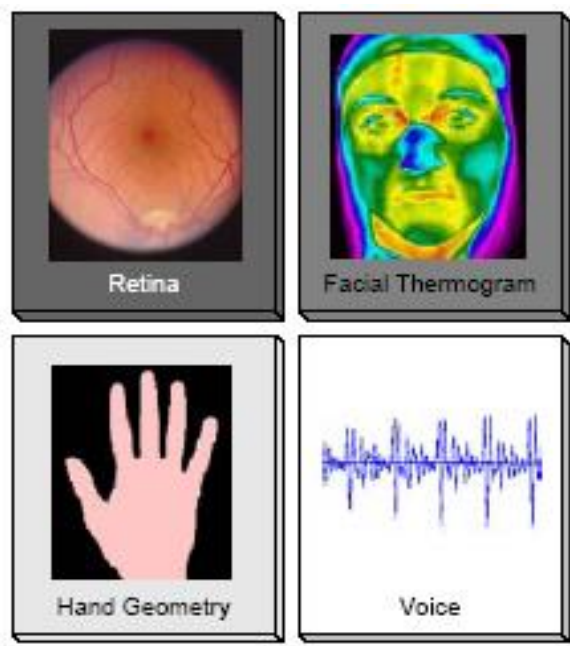

الثكل (2) أنمان

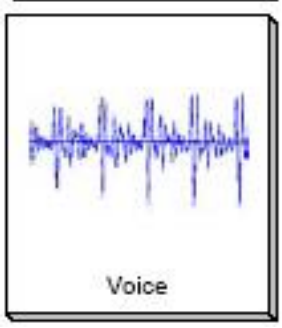

5. 5 5 5 ام التعرف على بصمة الإصبع

بداية ليكون النظام قادرًا على التعرف على شخص ما على أساس بصمته يحتاج - بالطبع- إلى مطابقة

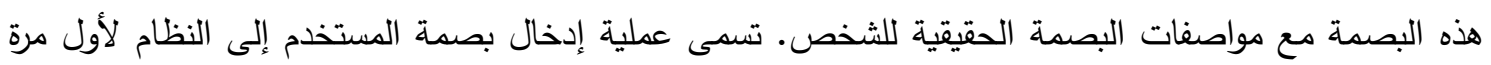
بالتسجيل، وفي هذه الحالة فإن سمات البصمة تخزن على شكل قالب في قاعدة البيانات[10].

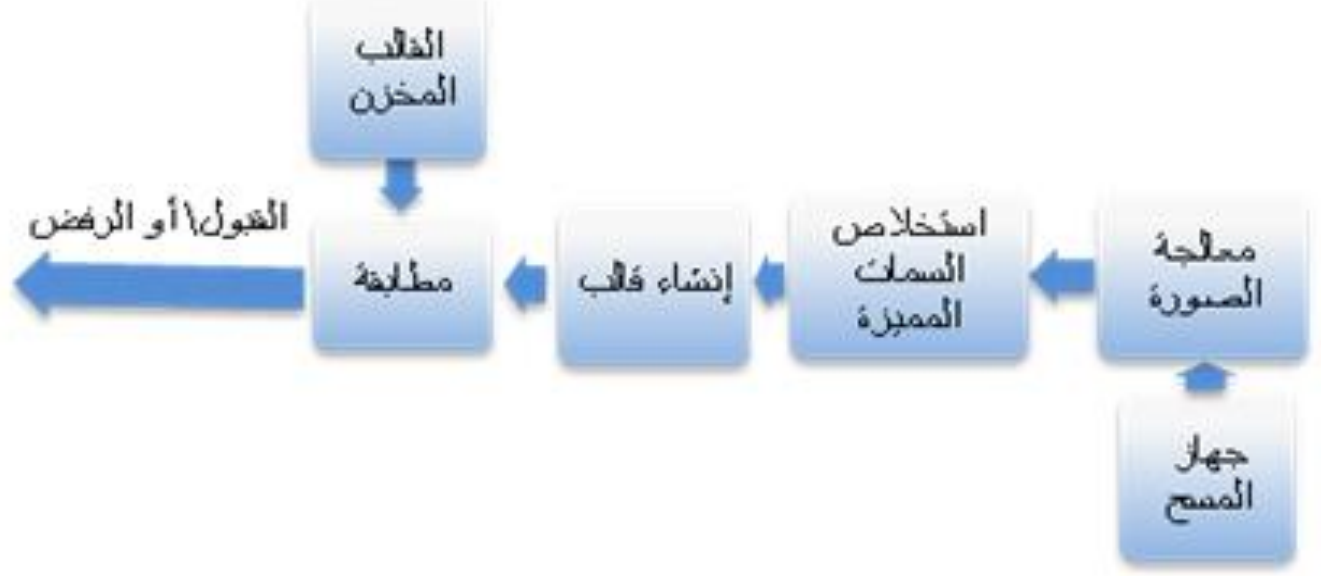

الثكل(3) مكونات نظام التعرف على بصمة الإصبع 
يقوم النظام بالتقاط صورة البصمة عن طريق الماسح. و ماسح بصمة الإصبع هو جهاز الكتروني يستخدم

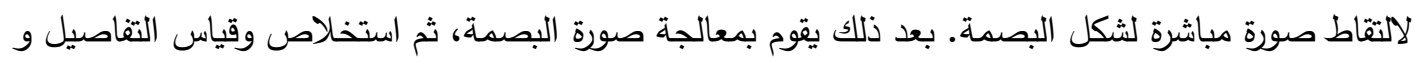

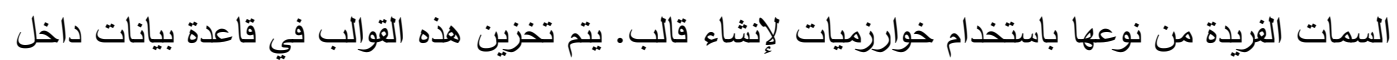
النظام،ويمكن تخزينها أيضا على بطاقة ذكية. يقوم المستخدم في كل مرة يحتاج فيها إلى تعريف شخصيته، بوضع إصبعه على الماسح، ينشئ النظام

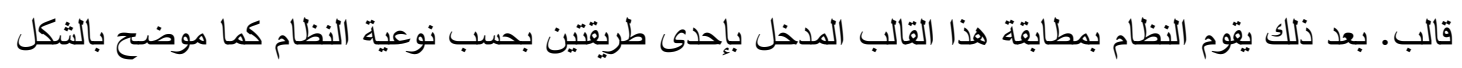

6. مصفوفة التواجد

عبارة عن تطبيق احصائي يستخدم لوصف نسيج الصورة المراد تمييزها من خلال استخلاص خواص الصورة عن طريق معادلات احصائية ،حيث يتم تحويل صورة بصمة الاصبع الى مصفوفة ثنائية الابعاد ويتم التعامل معها[11],[12] [12].

ان فكرة تكوين هذه المصفوفة تعتمد على قيم الازواج المتجاورة افقيا من النقاط في الصورة المراد تمييزها

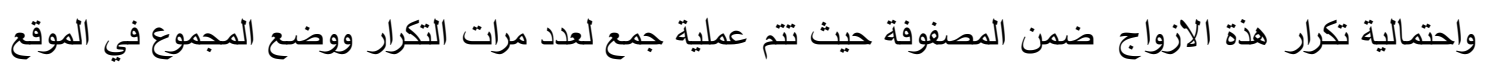

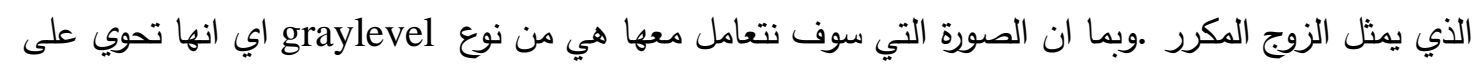

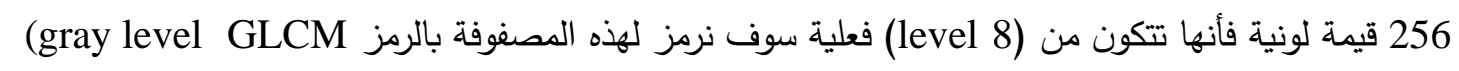
co-occurrence matrix)

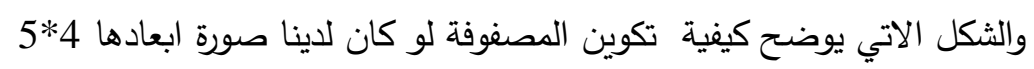

\begin{tabular}{|l|l|l|l|l|l|l|l|l|l|l|l|l|l|l|}
\hline 1 & 1 & 5 & 6 & 8 \\
\hline 2 & 3 & 5 & 7 & 1 \\
\hline 4 & 5 & 7 & 1 & 2 \\
\hline 8 & 5 & 1 & 2 & 5 \\
\hline
\end{tabular}

الثكل(4) كيفية تكوين المصفوفة

GLCM 1.6 استخلاص الصفات الخاصة بالمصفوفة

تحوي المصفوفة على مجموعة من الصفات الاحصائية التي تصف قيم الكثافة في نسيج الصورة ومنها يتم استخلاص الصفات الخاصة بنسيج الصورة [7] [13], والصفات التي تم استخدامها في هذا البحث لاستخلاص خصائص بصمة الاصبع هي: 
أ- التباين (contrast) وهو مقياس لثدة التباين بين كل نقطة ضوئية واخرى مجاورة لها بالنسبة لصورة البصمة كلها ,و يحسب عن طريق المعادلة التالية :

$F=\sum \sum(i-j)^{2} p(i, j)$

ب- الترابط (correlation) وهو مقياس لمدى ترابط كل نقطة ضوئية مع اخرى مجاورة لها بالنسبة للصورة باكملها , المعادلة التالية:

$\mathrm{F}=\sum \sum(\mathrm{i}, \mathrm{Mj}) *(\mathrm{j}-\mathrm{Mi}) * \mathrm{p}(\mathrm{i}, \mathrm{j}) / \sigma \mathrm{x} \sigma \mathrm{y}$.

ج-العزم (energy) وهوعبارة عن مجموع لمربع العناصرالموجودة في مصفوفة ال GLCM ويحسب وكما يلي:

$\mathrm{F}=\sum \sum \mathrm{p}(\mathrm{i}-\mathrm{j})^{2}$

د- التجانس(Homogeneity) يعطي مؤشر لكمية التجانس داخل النسيج ويحسب عن طريق المعادلة التالية : $\mathrm{F}=\sum \sum(\mathrm{p}(\mathrm{i}-\mathrm{j}) / \mathrm{i}+|\mathrm{i}+\mathrm{j}|)$

7. الشبكات العصبية الاصطناعية

يمكن القول إن تاريخ دراسة الشبكات العصبية الاصطناعية يعود الى عام 1943، اذ بني أول نموذج لخلية

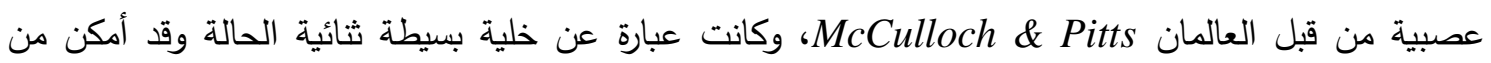
خلالها تمثيل الدوال المنطقية. وفي عام 1949 قدم العالم Hebb أول قاعدة لتعليم الشبكة العصبية أطلق عليها Hebbian learning Rule فالشبكات العصبية هي نوع من أنواع الذكاء الاصطناعي تقوم بتقليد طريقة عمل الدماغ البشري وخزن

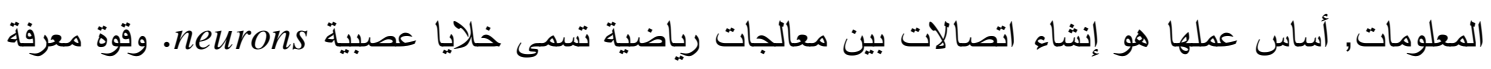

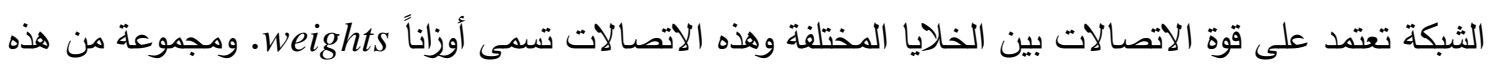
الخلايا العصبية تثكل طبقة وتسمى layer حيث تعمل خلايا هذه الطبقة على التوازي وفي نفس الوقت, وإن إن

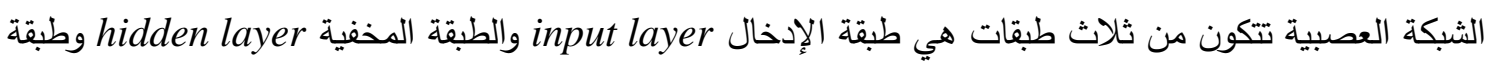
الإخراج output layer, ويتعلم النظام من خلال عملية تحديد عدد الخلايا العصبية في كل طبقة وكذلك ضبط أوزان الاتصالات بناء على بيانات التدريب. في مرحلة التدريب إذا احتاجت الثبكة العصبية لعلم مسبق لبيانات الإدخال والإخراج فإن مرحلة التعليم تسمى التعليم بُِعلم Supervised Training أما إذا لم تحتج الشبكة لبيانات

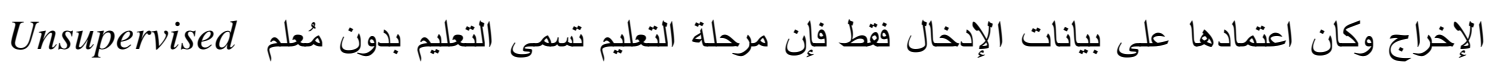
20] Training [3] [3الشكل رقم (5) يمثل الخلية العصبية الطبيعية. 


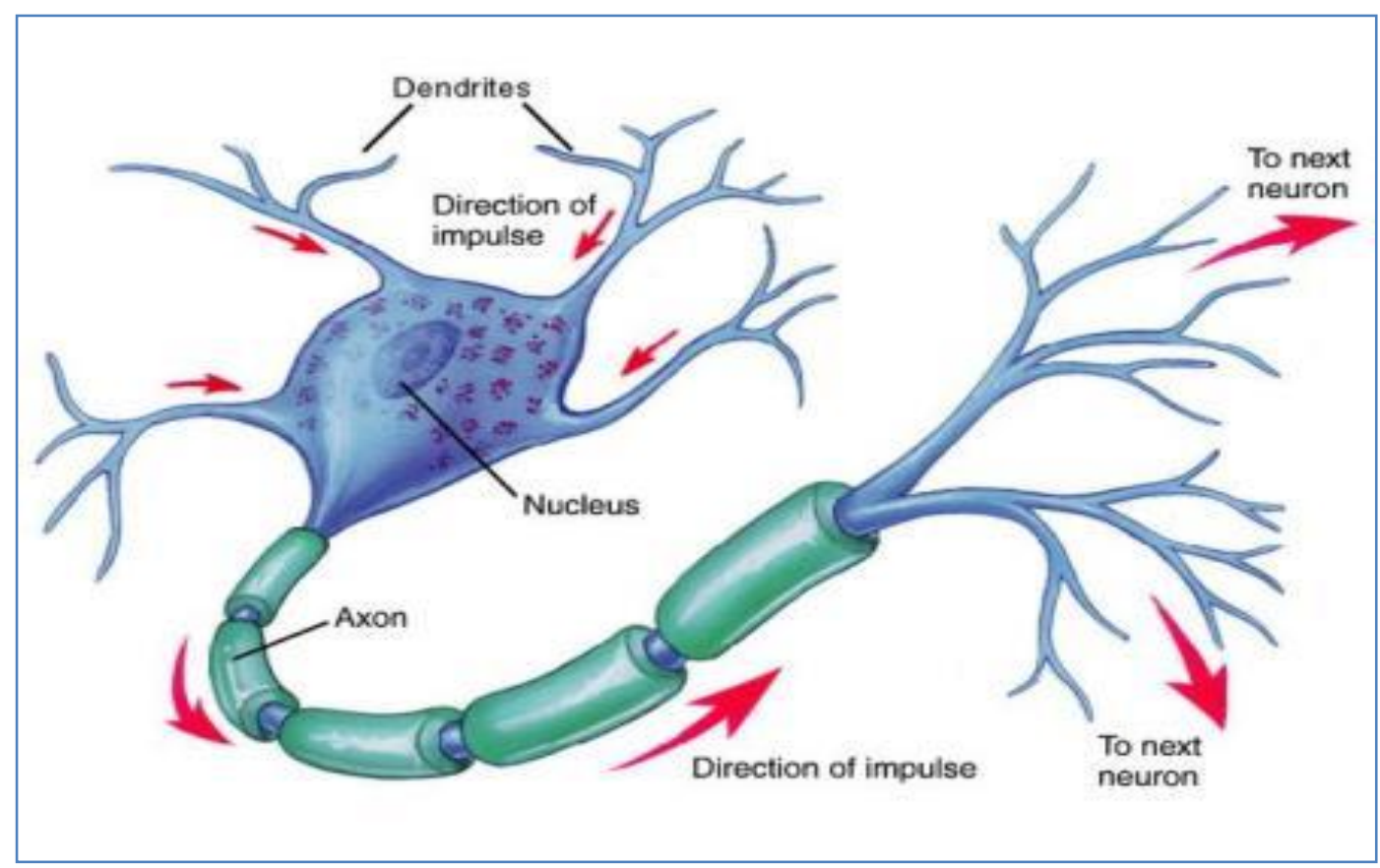

الثكل(5) الخلية العصبية الطبيعية

تم استخدام الثبكات العصبية الاصطناعية في عملية التمييزلما تقدمه من كفاءة عالية في العمليات الحسابية

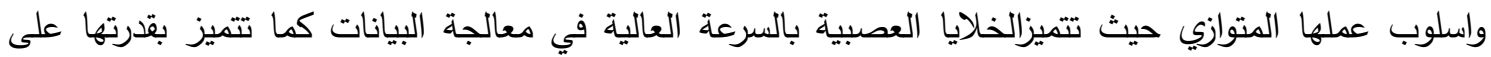
التعلم والتعامل مع انماط مختلفة من البيانات التي يكون جزء منها خاطئا مما جعلها مناسبة لكثيرمن التطبيقات مثل تمييز الصور والكلام ،تمييز الصوت الخ[6]. 8. وصف الثبكات العصبية الستخدمة في البحث

تم في هذا البحث استخدام ثلاث شبكات عصبية وهي الثبكة العصبية المتتالية (CNN) وشبكة دالة القاعدة الشعاعية(RBFN) وشبكة ايلمان وتطبيقها مع مصفوفة ال (GLCM) لتمييزبصمة الاصبع لشخص من بين مجموعة من الاشخاص التابعين لنفس قاعدة البيانات.

\section{8 الشبكات العصبية المتتالية}

شبكة الـ CNN مشابهة لشبكات التغذية الأمامية ولكن تحتوي على ترابط بالأوزان من طبقة الادخال الى كل طبقة تالية لها ومن كل طبقة الى الطبقات التالية لها. على سبيل المثال شبكة ذات ثلاث طبقات تملك ارتباطات من الطبقة 1 الى الطبقة 2 , ومن الطبقة 2 الى الطبقة 3, ومن الطبقة 1 الى الطبقة 3. وهذه الثبكة ايضاً تملك ارتباطات من الإدخال الى الطبقات الثلاثة كلها وهذه الارتباطات الإضافية قد تحسن من سرعة تعليم الشبكة للإخراج المرغوب [14],[15]. إن شبكة الذكاء الاصطناعي CNN مشابهة للشبكات العصبية ذات التغذية الأمامية FFNN في أنها تستخدم خوارزمية الانتشار خلفاً لتعديل الأوزان ولكن العلامة الفارقة الأساسية لهذه الثبكة هي أنَ كل طبقة من العقد تكون مترابطة مع كل الطبقات السابقة من العقد, وتستخدم هذه الشبكة دالة التحويل Tan-sigmoid أو log - للوصول الى الحالة الامثل[3],[12] . والثكل (6) يبين الهيئة

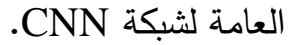




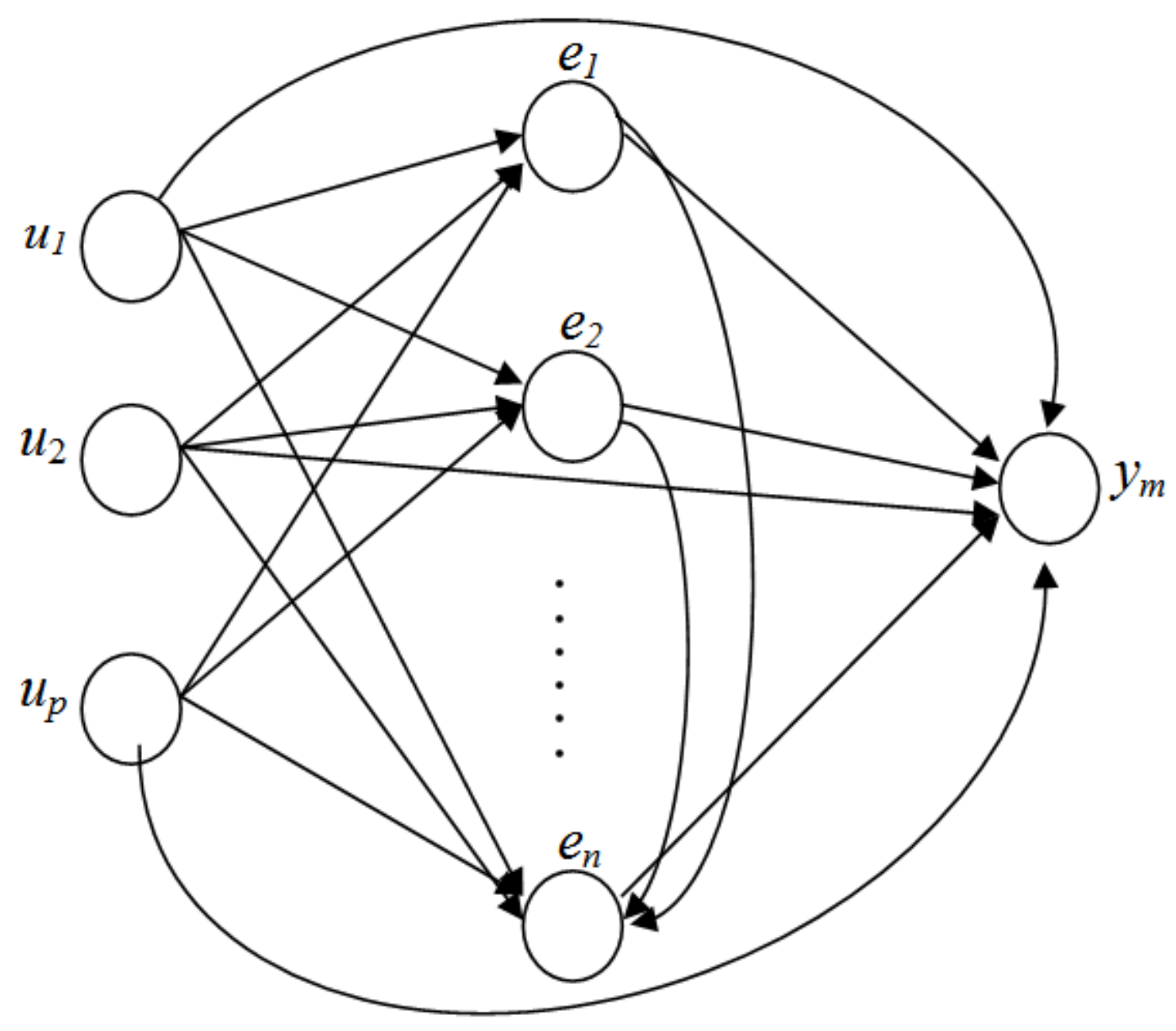

الثكل (6) الهيئة العامة لشبكة CNN

الشبكات العصبية المتتالية تحوي على ثلاث طبقات : طبقة الادخال , والطبقة المخفية وطبقة الاخراج . والتي هي ,p,., , , CNN 1.1.8 خوارزمية شبكة الـ

إن معالجة التدريب لشبكة CNN هي معالجة تكرارية iterative process وكل تكرار iteration يتكون من عدد من الخطوات. وإن تدريب الشبكات العصبية المتتالية يمكن أن يتم عن طريق الانتشار خلفاً [16]. وفيما يلي الخطوات الأساسية: 1- في البدء تهيأ الاوزان الابتدائية، وذلك باختيار قيم ابتدائية صغيرة وعلى نحو عشوائي لأوزان الخلايا كلها في الثبكة العصبية . 2- اختيار زوج التدريب من مجموعة التدريب [ Input, Target ], ونحسب قيمة الإخراج لكل وحدة j في كل

طبقة L المكا مبين في المعادلة (5) والمعادلة (6). فالدعادلة التي تصف اخراج الطبقة المخفية هي:

$\mathrm{e}_{\mathrm{i}}=\varnothing\left(\sum_{\mathrm{j}=1}^{\mathrm{p}} \mathrm{W}_{\mathrm{ij}}^{\mathrm{eu}} \mathrm{u}_{\mathrm{j}}+\sum_{\mathrm{k}=1}^{\mathrm{i}-1} \mathrm{~W}_{\mathrm{ik}}^{\mathrm{ee}} \mathrm{e}_{\mathrm{k}}+\mathrm{b}_{\mathrm{i}}^{\mathrm{e}}\right)$ 
(الثي

هما الاوزان القابلة للتعديل. $W_{i j}^{e u}, W_{i k}^{e e}$

u $u_{j}, e_{k}$

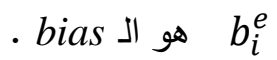

والدالة التي تصف طبقة الاخراج تكون دالة خطية بحتة وهي:[15]

$\mathrm{y}_{\mathrm{i}}=\left(\sum_{\mathrm{j}=1}^{\mathrm{p}} \mathrm{W}_{\mathrm{ij}}^{\mathrm{yu}} \mathrm{u}_{\mathrm{j}}+\sum_{\mathrm{k}=1}^{\mathrm{n}} \mathrm{W}_{\mathrm{ik}}^{\mathrm{ye}} \mathrm{e}_{\mathrm{k}}+\mathrm{b}_{\mathrm{i}}^{\mathrm{y}}\right)$

حيث

祘

هما الاوزان القابلة للتعديل. $W_{i j}^{y u}, W_{i k}^{y e}$

u هما الاخراج من عقد الادخال والعقد المخفية (الوسطى) على التتابع. $u_{j}, e_{k}$

. bias هو الإخان

3- نحسب الخطأ بين الإخراج الحقيقي للشبكة والإخراج المطلوب من زوج التدريب، ثم نستخدم القيم المحسوبة من

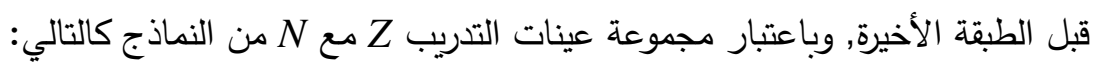
(7) $E(Z ; w)=\sum_{i=1}^{N}\left(\left(y_{i}(j)-T_{i}(j)\right)^{2}\right)$

حيث

N هي عدد النماذج. w هو الوزن القابل للتعديل. 䒠

.

4- تحديث الأوزان باستخدام المعادلة الآتية مع الأخذ بنظر الاعتبار منحني التعديل , فان الخوارزمية التكرارية

$$
\left\{\begin{array}{l}
\mathrm{w}^{(\mathrm{i}+1)}=\mathrm{w}^{(\mathrm{i})}-\mu^{(\mathrm{i})} \mathrm{H}^{(\mathrm{i})} \mathrm{E}^{\prime}\left(\mathrm{w}^{(\mathrm{i})}\right) \\
\mathrm{w}^{(0)}=\mathrm{w}_{0}
\end{array}\right\}
$$

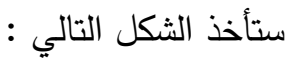

ب

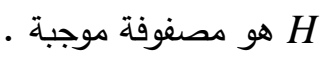

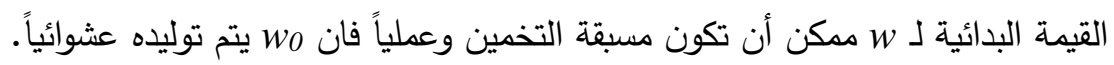
وفيما يتعلق بالمعادلة (8) فان تحديث منحني التعديل يبنى ضمنياً على اشتقاق دالة الكيلة الكلفة مع الأخذ بنظر الاعتبار منحني التعديل [16].

5- نرجع إلى الخطوة الثانية ونكررها لكل النماذج p والى أن يصل الخطأ إلى قيمة مقبولة.من (5) و(6) اذا تم

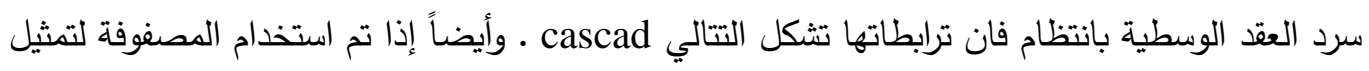
الترابطات بين العقد الوسطى فان المصفوفة سوف تكون مصفوفة ثلاثية منخفضة أو مصفوفة ثلاثية علوية

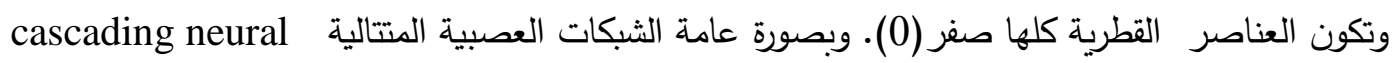
network 
الثكل (6) فكل عقد الادخال والعقد الوسطية تكون مترابطة مع الإخراجات وكل العقد الوسطية تكون مترابطة مع بعضها [16]

RBFN 2.8. شبكة دالة القاعدة الشعاعية

تعتبر RBFN من شبكات التغذية الأمامية feed forward وتحتوي على طبقة مخفية واحدة ودالـة

اللياقة لهذه الطبقة تسىى basis functions والثكل(7) يمثل الهيكلية العامة لشبكة دالة القاعدة الثعاعية [4].

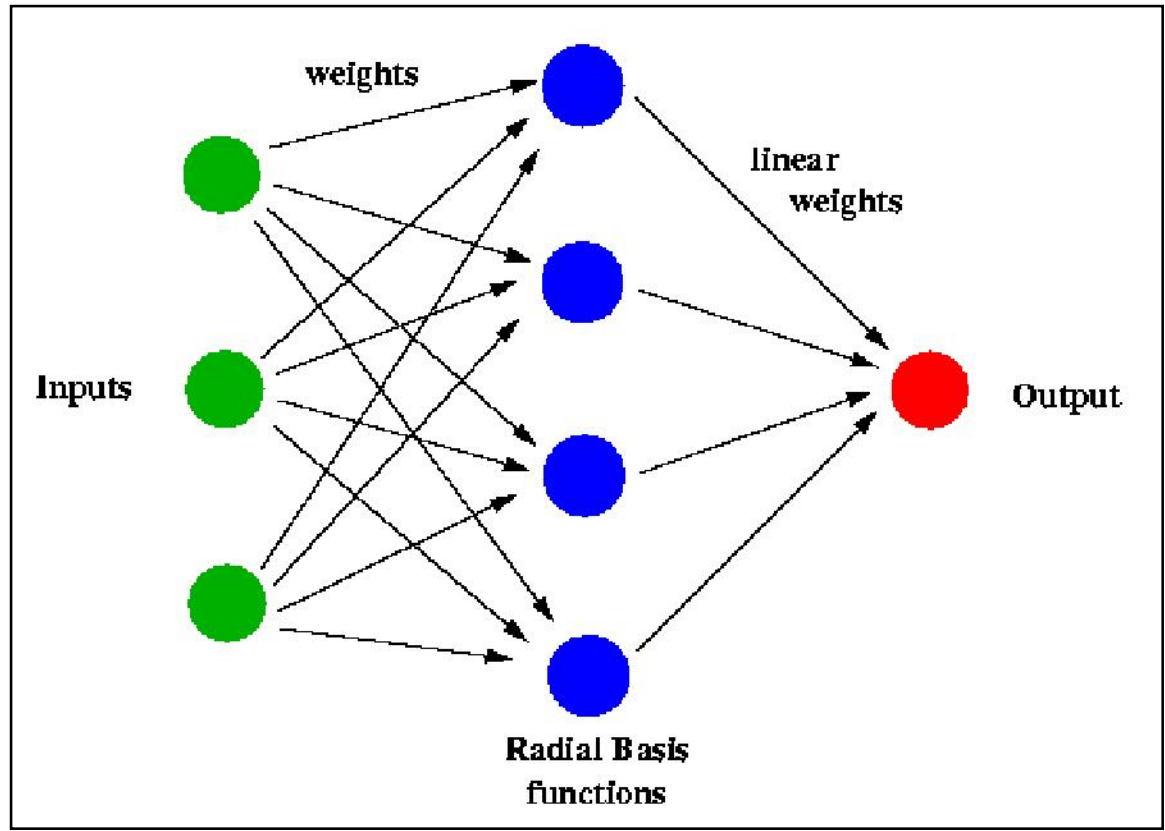

الثكل (7): الهيكلية العامة لالة القاعدة الثعاعية

تقوم هذه الثبكة بتحويل المدخلات بطريقة غير امطية ثم إيجاد المنحني المناسب

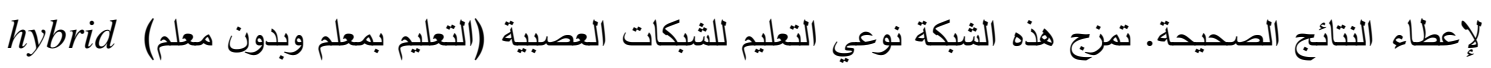
of unsupervised and supervised learning تعليم بدون مُعَلم unsupervised ويتم عنقدة البيانات إلى مجاميع حسب قانون المسافة الإقليدية بين بيانات

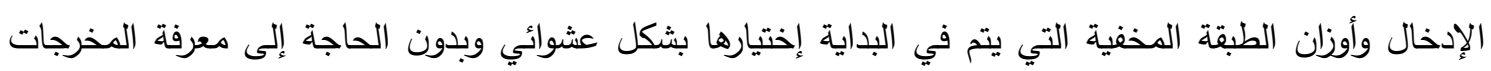
وتسمى دالة اللياقة الخاصة بهذه الطبقة Gaussian radial basis functions, أما التعليم بين الطبقة المخفية وطبقة الإخراج فيكون تعليماً بِمُعلم supervised ويعتمد على نسبة الخطأ بالاعتماد على المخرجات. المزايا الرئيسة لشبكة دالة القاعدة الثعاعية هي بساطة الدالة المستخدمة ومنحني الدالة يكون سلساً جداً وشعاعي التتاظر

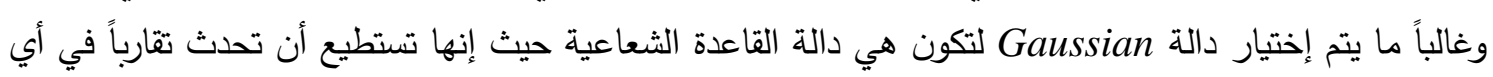
وظيفة ثابتة من دون الاعتماد على نموذج النظام[5]. وتمثل Gaussian بين طبقة الإدخال والطبقة المخفية بالمعادلة التالية: $\varphi(x)=\exp \left(-\frac{\left\|x-c_{j}\right\|^{2}}{2 \sigma^{2}}\right)$

حيث c يمثل الوزن المرتبط بالطبقـة المخفية ز . 
o

أما عملية المعالجة بين الطبقة المخفية وطبقة الإخـراج فتكون خطية ويتم حساب ناتج طبقة الإخراج كالتالي : $y_{m}=\sum_{j=1}^{m} w_{j} \varphi_{j}$

حيث w $w_{j}$ هو وزن الاتصال بين الطبقة المخفية ز وطبقة الإخراج. m هو عدد الخلايا العصبية في الطبقة المخفية.

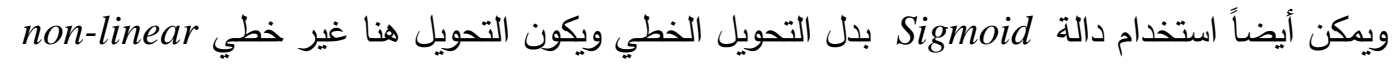

ثم يتم تعديل أوزان طبقة الإخراج بالمعادلة التالية والتي تسمى linear least estimation : $n e w_{-} w=$ old $_{-} w+\left(E^{*}\right.$ learning_rate $\left.*\right)$

حيث إن learning _ rate هي نسبة التعلم وتكون بين [ 0.0 - 1.0 ] ـ و E هي نسبة الخطأ

وتُسمى دالة الأداء performance function

$E(k)=\frac{1}{2}\left(y(k)-y_{m}(k)\right)^{2}$

حيث y يمثل الإخراج الحقيقي للشبكة و y $y_{m}$ هو ناتج طبقة الإخراج. وكلما اقتربت نسبة الخطأ من الصفر ازدادت فرصة الوصول للحل الأمثل.

إن RBFN تجهز بحل ممتاز للعديد من مشاكل تمييز الأنماط والتصنيف والتتبؤ. وكذلك RBFN مدكن

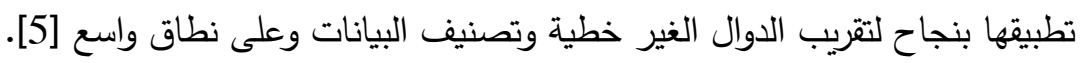

1.2.8 خوارزمية شبكة دالة القاعدة الثعاعية RBFN

عملية التدريب للا RBFN هي عملية تكرارية أو دورية. وكل دورة تتألف من الخطوات

التالية [5]:

1- تكوين أوزان الثبكة W بقيم عشوائية صغيرة.

2- اختيار زوج التدريب التالي من مجموعة التدريب [input, target], وحساب قيم الإخراج باتجاه أمامي لكل وحدة ز من الطبقة المخفية وذلك:

$\varphi_{j}(x)=\exp \left[-\frac{\left\|x-\mu_{j}\right\|^{2}}{2 \sigma_{j}^{2}}\right]$

3- حساب قيم الاخراج وباتجاه أمامي لكل وحدة k من طبقة الإخراج وذلك:

$\psi_{k}(x)=\sum_{j=1}^{L} W_{j k} \varphi_{j}(x)+W_{0}$

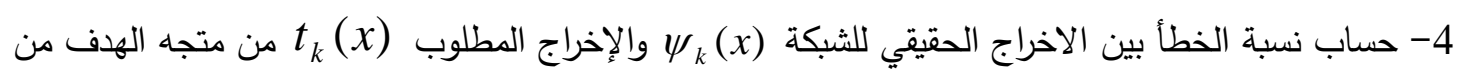
زوج التدريب, وبعدها, استخدام قيم (x) المحسبة

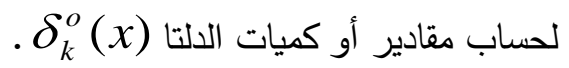




$$
\delta_{k}^{o}(x)=\left(t_{k}(x)-\psi_{k}(x)\right)
$$

$W_{j k}^{\text {new }}=W_{j k}^{\text {old }}+\Delta W_{j k}$

$\Delta W_{j k}=\eta \delta_{k}^{o}(x) \varphi_{j}(x)$

6- العودة للخطوة 3 واعادة الخطوات لكل نموذج x في مجموعة التدريب لحد الوصول إلى نسبة الخطأ الكلية والقيمة المقبولة.

3.8. - 3.8

تتألف شبكة إيلمان Elman في الأقل من ثلاث طبقات من الخلايا: طبقة الإدخال, والطبقة الوسطى الطي الطبان

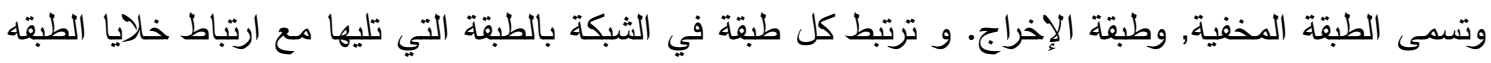

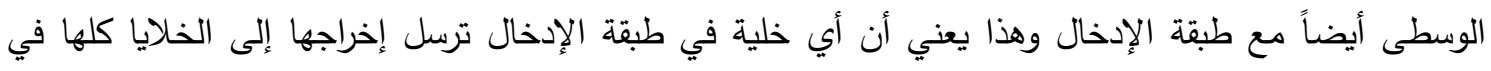

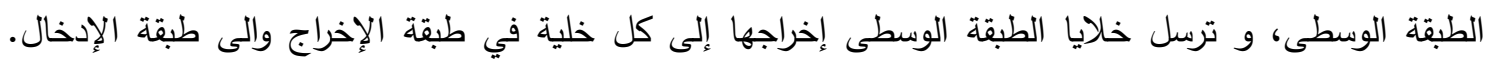

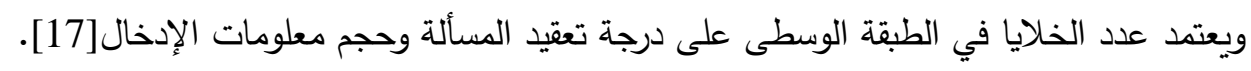

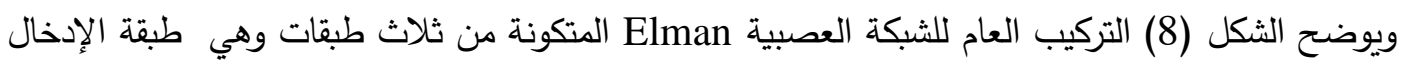

\section{والطبقة الوسطى وطبقة الإخراج على الترتيب.}

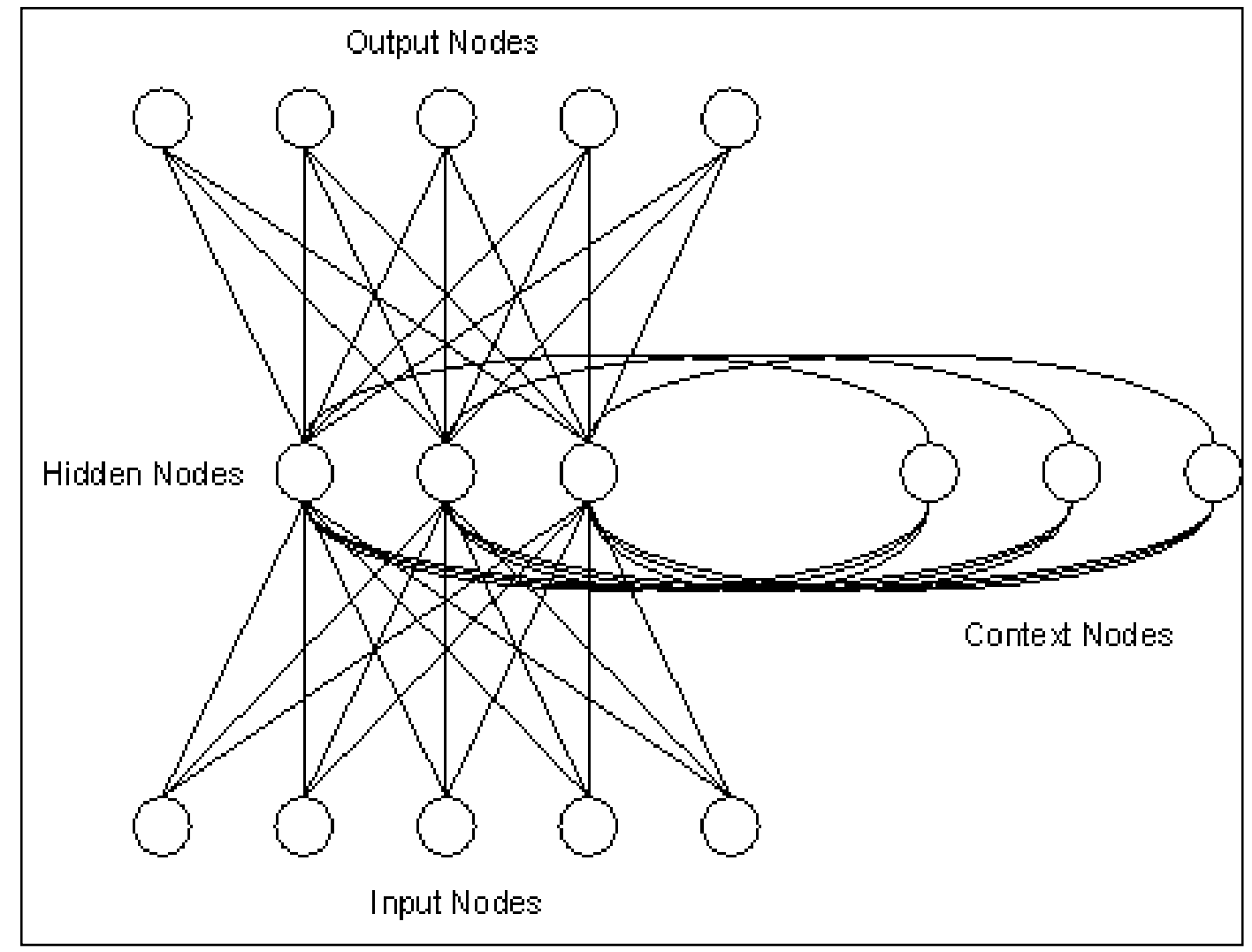

الشكل(8) التركيب العام للشبكة العصبية إيلمان ENN 
1.3.8

إن معالجة التدريب لثبكة ENN هي معالجة تكرارية وكل تكرار يتكون من عدد من الخطوات ـ ويضرب

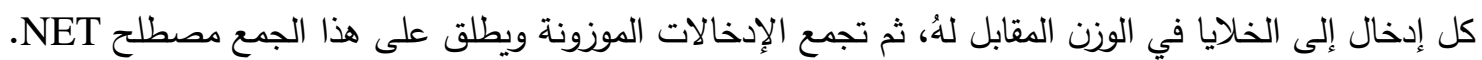
وبعد حساب الإخراج NET يمرر على دالة F المتمثلة بإحدى دوال الفعالية لتحويلها إلى الثارة

الـ [17] Out (17)

وقد استخدمت في هذا البحث الدالة السيجماوية Sigmoid Function إذ توصف بأن مشتقتها بسيطة .

$$
o u t=f\left(N E T_{j}\right)=\frac{1}{1+e^{-N E T j}}
$$

$f^{\prime}\left(N E T_{j}\right)=$ out $(1-$ out $)$

Squashing أو دالة الإخماد logistic Function وتسمى الدالة السجماوية أحياناً بالدالة المنطقية Function

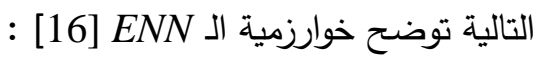
1- في البدء تهيأ الأوزان الابتدائية، وذلك باختيار قيم ابتدائية صغيرة وعلى نحو عشوائي لأوزان الخلايا كلها في

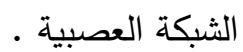
2- اختيار زوج التدريب من مجموعة التدريب [Input ، Target] الذي يمثل الإدخال والإخراج المطلوبين وتحسب قيم الإخراج لكل خلية في كل طبقة Lي في الثبكة. net $_{\mathrm{pj}}^{\mathrm{L}+1}=\sum_{\mathrm{i}=1}^{\mathrm{n}} \mathrm{W}_{\mathrm{ij}}^{\mathrm{L}}$ out $_{\mathrm{i}}^{\mathrm{L}}$

$$
\text { out }_{p j}^{\mathrm{L}+1}=f\left(\text { net }_{\mathrm{pj}}^{\mathrm{L}+1}\right)=\frac{1}{1+\mathrm{e}^{- \text {net }_{\mathrm{pj}}^{\mathrm{L}+1}}}
$$

net $t_{p j}^{L+1}$

وان out كما أن out ${ }_{p j}^{o}=x_{j}$ 3- يحسب الخطأ بين الإخراج الحقيقي للشبكة والإخراج المتوقع من زوج التدريب. ثم نستخدم out

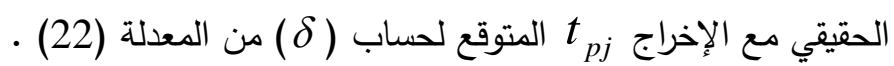
$\delta_{p j}^{o}=\left(t_{p j}-o u t_{p j}^{o}\right) f^{\prime}\left(n e t_{p j}^{o}\right)$

4- نحسب قيمة ( $\delta$ ) للطبقات الوسطى كلها باستخدام الخطأ خلفاً وحسب

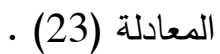

$$
\delta_{p i}^{L+1}=f^{\prime}\left(\text { net }_{p i}^{L+1}\right)\left[\sum_{j=1}^{m^{L+2}} \delta_{p j}^{L+2} w_{i j}^{L+1}\right]
$$




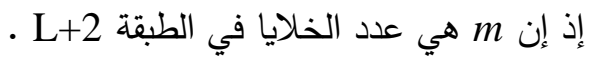

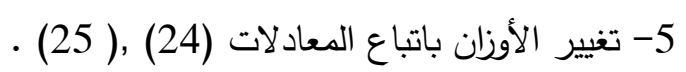

$$
\begin{aligned}
& w_{i j}^{\text {new }}=w_{i j}^{\text {old }}+\Delta w_{i j}^{L} \\
& \text { إذ إن } w_{i j}^{L} \text { يحسب من المعادلة (20) }
\end{aligned}
$$$$
\text { ...(25) } \Delta w_{i j}^{L}=\eta \delta_{p j}^{L+1} \text { out } t_{p j}^{L}
$$

6- تغيير الأوزان للطبقات المخفية, عكس طبقة الإخراج فان قيمة الخطأ لا يمكن حسابها مباشرة, حيث لا

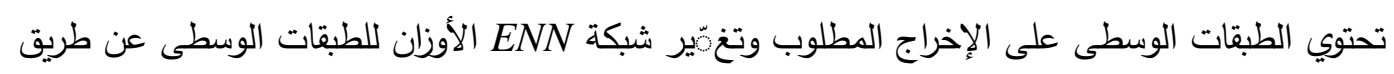

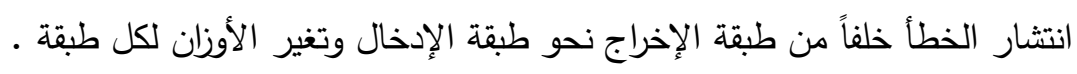

$$
\text { 9. مراحل العمل المستخدمة في البحث }
$$

في هذا البحث تم تمييز بصمة الاصبع بالاعتماد على مجموعة بيانات مزودة لطبقة الإدخال الخاصة

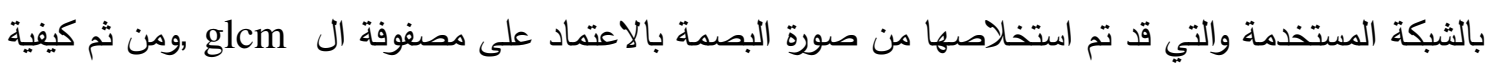
تدريب هذه البيانات وأخيرا كيف يتم اختبار الثبكة المستخدمة في التميز وكما هو موضح بالثكل (8) الذي يوضح خطوات سير العمل. وعلى هذا الأساس فان عملنا هذا يبدأ بتحضير بيانات الإدخال ومعالجتها، ثم اختيار

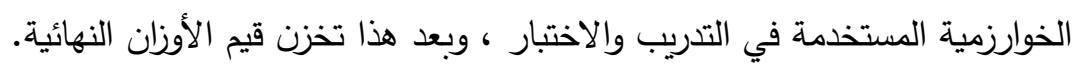

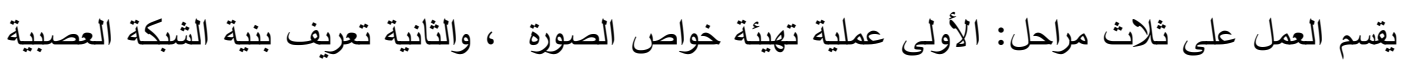
المستخدمة بالاعتماد على الخواص المستخلصة من مصفوفة ال glcm والثالثة هي عرض نتيجة التمييز وتحديد هوية الثخص من قاعدة البيانات أو رفضه.

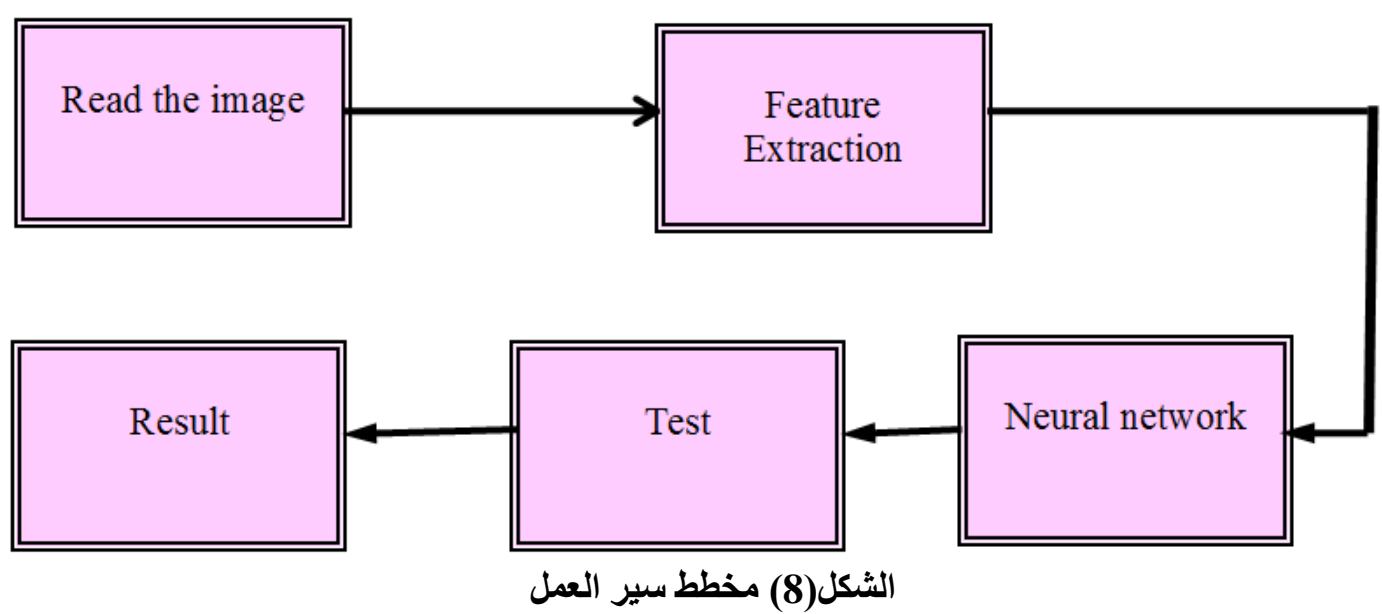

1.9. تهيئة البيانات المدخلة

في البداية يتم ادخال صورة بصمة الاصبع باسلوب معين مثلا بواسطة الماسح الضوئي اوصورة مخزونة على في

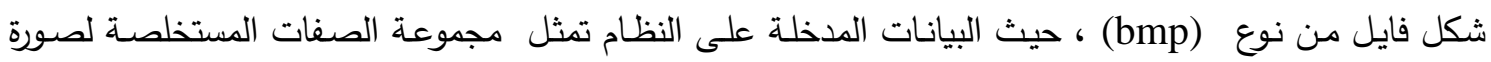
بصمة الاصبع , التي تم الحصول عليها من خلال دراسة وتحليل الصورة باستخدام مصفوفة (GLCM) حيث يتم

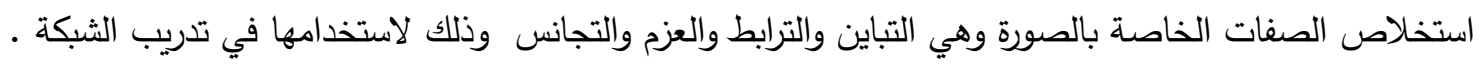


2.9. تهيئة الثبكة العصبية المستخدمة

إن هيكلية الثبكة يجب أن تتلاءم مع هيئة المشكلة المراد حلها ، حيث من خلال العمل تبين ان الشبكة تحتاج الى (4) عقد تمثل متجه الإدخال (Input Vector) التي تمثل الصفات الناتجة من تحليل الصورة باستخدام مصفوفة التواجد (GLCM) و(6) عقد تمثل قيمة الاخراج للشبكة (output vector) التي تكون كافية لكي تلئي

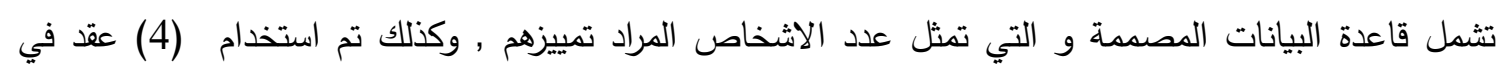
- الطبقة المخفية (hidden layer)

تم في هذا البحث استخدام الثبكات العصبية الثلاث (CNN, RBFN, ELMN) R

3.9. عملية التمييز

بعد أن تم تدريب الثبكات وأصبحت جاهزة للعمل كأداة في نظام تمييز بصمة الاصبع يكون عملها

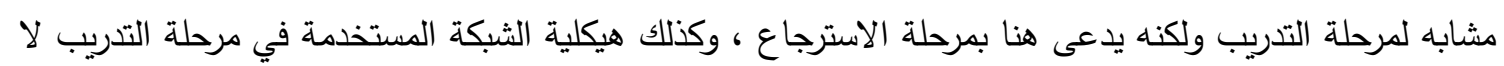

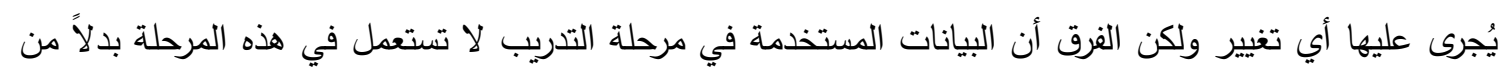

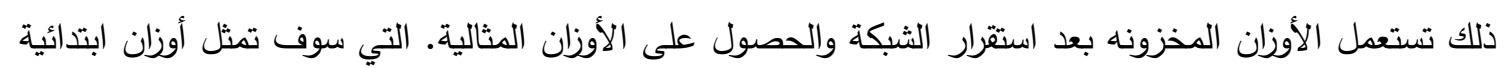
وثابتة في الثبكة ، في هذه الحالة تستطيع الثبكة تحليل الإدخال لكي تميز صورة البصمة من خلال صفات التان

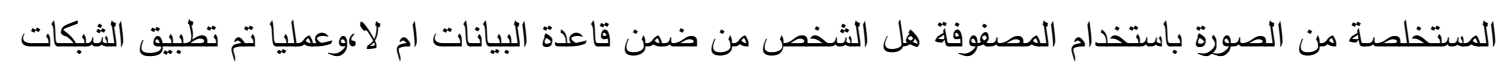
الثلاث على (6)اشخاص لكل شخص(3) عينات(بصمات) وبذلك يصبح عدد العينات الددربة هو (18)عينة، وكل فئل عينة تمثل ب(4) قيم هي خواص صورة البصمة التي تم استخلاصها (3) علئات 10. حساب مقاييس التقييم

سوف يتم في هذا البحث استخدام نوعين من مقاييس التقييم لاختبار كفاءة كل شبكة المقياس الاول هو training rate (26) $\% 100 \times$ نسبة صحة التصنيف =

$$
\text { العدد الكلي للبصمات }
$$

اما المقياس الثاني فهو recognition rate الذي يستخدم لقياس نسبة تمييز الثبكة حيث تعرف الاخطاء

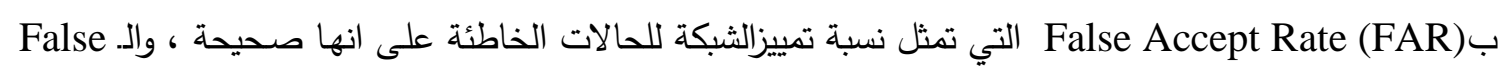
Reject Rate (FRR) القانون التالي(19):

$$
\begin{gathered}
F A R=\frac{\text { Number of incidents of false accepted }}{\text { Total number of samples }} \times 100 \% \\
F R R=\frac{\text { Number of incidents of false rejected }}{\text { Total number of samples }} \times 100 \%
\end{gathered}
$$

حيث ال RR يحسب بالثكل التالي :

$R R \%=100-(F A R+F R R)$ 
11. نتائج عملية تمييز بصمة الاصبع

في هذا العمل تم استخدام الصور ذات التدرجات الرمادية ،حيث تم تمييز بصمة الاصبع باستخدام ثلاث شبكات عصبية وبالاعتماد على (GLCM) حيث كانت قاعدة البيانات مؤلفة من مجموعة من الاشخاص المراد

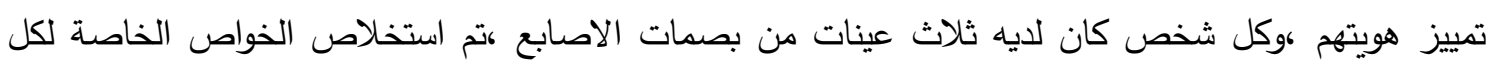

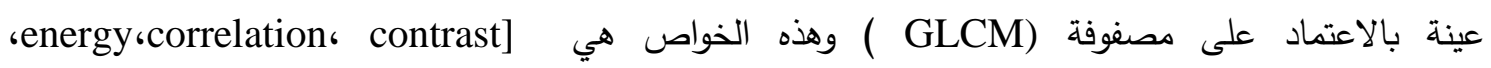

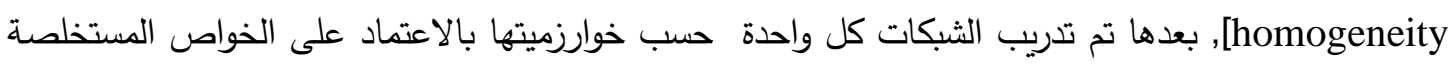

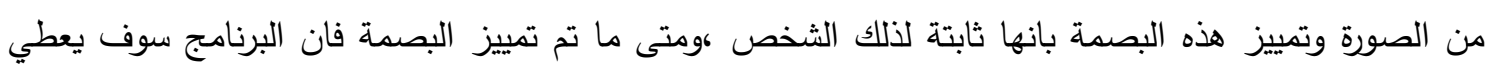

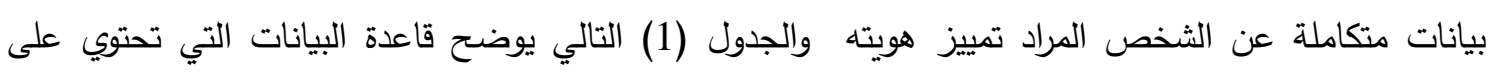
العينات الخاصة بكل شخص مع حساب الخواص الثابتة لكل عينة بالاضافة الى معلومات خاصة بذلك الثخص

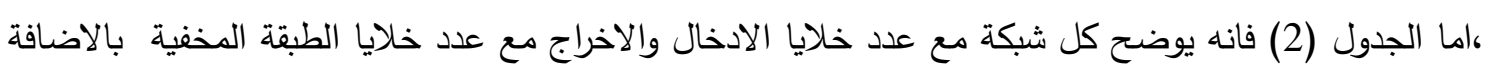
الى قيم مقاييس التقييم لكل شبكة,اما عددعينات التديب فكانت مكونة من(18) صورة بصمة اصبع وعددعينات الاختبار ( 3 )بصمات تابعة لاشخاص مختلفين.

جدول(1) المعلومات الخاصة بقاعدة البيانات

\begin{tabular}{|c|c|c|c|c|c|c|c|}
\hline التجانس & العزم & الارتباط & التباين & عينة البصمة & العمر & الجنس & الاسم الكامل \\
\hline 0.9256 & 0.2165 & 0.915 & 0.1488 & & 30 & ذكر & احمد محمد عمر \\
\hline 0.9145 & 0.2047 & 0.904 & 0.1711 & & & & \\
\hline 0.9347 & 0.2307 & 0.9258 & 0.1306 & & & & \\
\hline 0.8998 & 0.1973 & 0.8827 & 0.2004 & & 38 & ذكر & اوس علي احمد \\
\hline 0.8833 & 0.1894 & 0.8526 & 0.2337 & & & & \\
\hline 0.9047 & 0.2378 & 0.8630 & 0.1905 & & & & \\
\hline 0.9129 & 0.3013 & 0.8036 & 0.1741 & & 25 & انثى & نور غانم علي \\
\hline
\end{tabular}




\begin{tabular}{|c|c|c|c|c|c|c|c|}
\hline 0.8928 & 0.2133 & 0.8459 & 0.2145 & & & & \\
\hline 0.8089 & 0.2505 & 0.8148 & 0.2043 & & & & \\
\hline 0.9058 & 0.2834 & 0.7916 & 0.1883 & & 33 & ذكر & احمد حازم صـابر \\
\hline 0.898 & 0.2672 & 0.8072 & 0.2041 & & & & \\
\hline 0.8991 & 0.2741 & 7975 & 0.202 & & & & \\
\hline 0.7161 & 0.0991 & 0.6454 & 0.8129 & & 45 & ذكر & يونس بلال عمار \\
\hline 0.6946 & 0.0703 & 0.6993 & 0.9535 & & & & \\
\hline 0.721 & 0.1018 & 0.5746 & 0.7886 & & & & \\
\hline 0.8914 & 0.3444 & 0.7565 & 0.2238 & & 27 & انثى & سوسن عماد سعيد \\
\hline 0.8769 & 0.3538 & 0.5011 & 0.2501 & & & & \\
\hline 0.872 & 0.3488 & 0.4814 & 0.2593 & & & & \\
\hline
\end{tabular}

جدول (2) عدد الخلايا في طبقة الادخال والاخراج والطبقة المخفية وعدد التكرارات لكل شبكة مع حساب مقياس TR,RR

\begin{tabular}{|c|c|c|c|c|c|c|}
\hline $\begin{array}{c}\text { Training } \\
\text { Rate }\end{array}$ & $\begin{array}{c}\text { Recognition } \\
\text { rate }\end{array}$ & $\begin{array}{c}\text { iteration } \\
\text { number }\end{array}$ & $\begin{array}{c}\text { Nodes of } \\
\text { hidden } \\
\text { layers }\end{array}$ & $\begin{array}{c}\text { Nodes } \\
\text { of } \\
\text { output } \\
\text { layers }\end{array}$ & $\begin{array}{c}\text { Nodes of } \\
\text { input } \\
\text { layers }\end{array}$ & $\begin{array}{c}\text { Neural Network } \\
\text { Name }\end{array}$ \\
\hline$\% 100$ & $\% 100$ & 18 & 4 & 6 & 4 & RBFN \\
\hline$\% 100$ & $\% 99.877$ & 2382 & 4 & 6 & 4 & ELMAN \\
\hline$\% 100$ & $\% 99.542$ & 4158 & 4 & 6 & 4 & CNN \\
\hline
\end{tabular}


من خلال دراسة عملية التمييز لصور بصمة الاصبع باستخدام الشبكات العصبية الاصطناعية وبالاعتماد

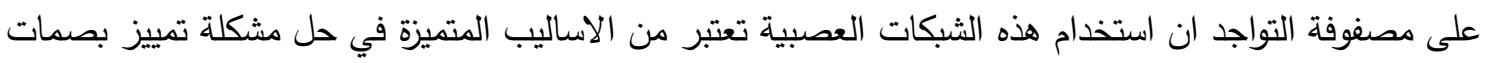
الاصابع حيث اعطت نتائج ممتازة عند التمييز على عدة عينات لبصمات الاصابع مع العلم ان الثبكة العصبية

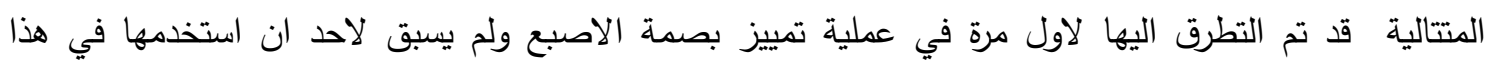

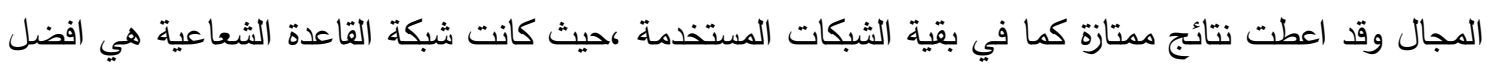
الثبكات المستخدمة من خلال ملاحظة قيم مقاييس التقييم المستخدمة في هذا البحث،ففي مرحلة التدريب كانت

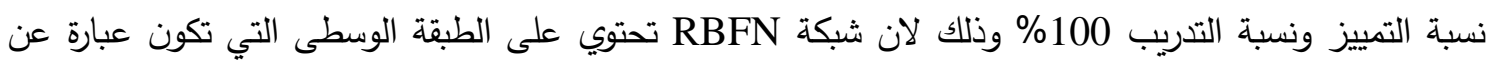

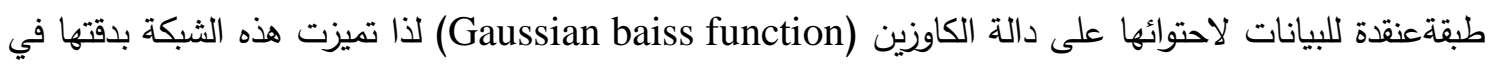

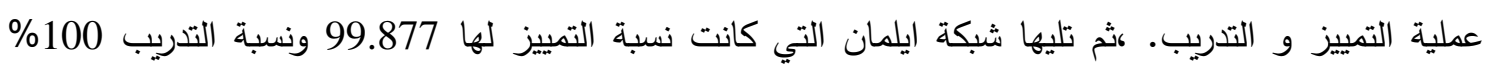

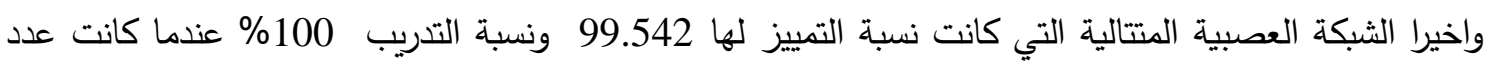
العقد في طبقة الادخال (4) عقد , عدد العقد في الطبقة المخفية (4) عقد, عدد العقد في طبقة الاخراج(6) عقد بالنسة للثلاث شبكات.

عند استخدام التقنيات الذكائية المتمثلة بالثبكات العصبية الثلاث التي تم استخدمها ضمن البحث اعطت نتائج ممتازة وبذلك كانت الطرق الذكائية افضل بكثيرمن الطرق التقليدية في تمييز بصمة الاصبع حيث الاخيرة لاتستطيع اعطاء نسبة تمييزاعلى من جيدة، حيث صفة التقنيات الذكائية انها تعطي تصنيفات ممتازة . 


\section{المصادر}

سعيد ،نغم ثروت ، (2006) ، "تمييز بصمة الاصبع باستخدام البعد الكسري "، رسالتماجستير،

قسم علوم الحاسبات ، كلية علوم الحاسبات والرياضيات،جامعة الموصل ، العراق.

النعيمي، ميسون خضر،(2011)،"تمييز بصمة الاصبع باستخلاص الخواص وباعتماد الثبكات

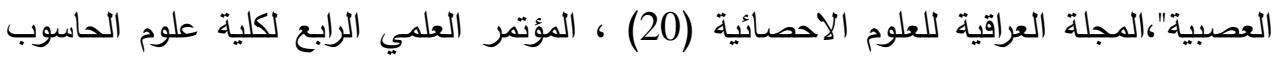

$$
\text { والرياضيات ص [ [690-975]،جامعة الموصل،العراق العرديه }
$$

قبع، اسراء زهير مجيد , (2012), (تخمين مشاريع البرمجيات باستخدام اساليب الذكاء

الاصطناعي", رسالة ماجستير، قسم هندسة البرمجيات ، كلية علوم الحاسوب والرياضيات،جامعة

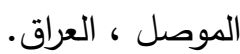

رشيدلي ،كرم محمد مهدي صالح , (2012),"تصميم وتتفيذ نظام كثف التطفل باستخدام التقنيات

الذكائية ", رسالة ماجستير، قسم هندسة البرمجيات ، كلية علوم الحاسوب والرياضيات،جامعة

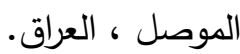

خليل ،شهباء ابراهيم, (2006), " الاسترجاع الكفوء للوسائط المتعددة المعتمد على المحتوى

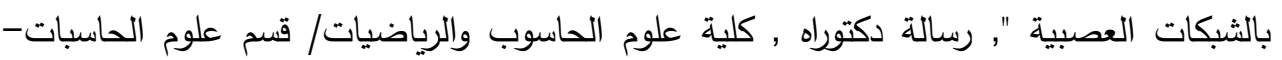
جامعة الموصل.

الزبيدي ،لكيب محمد ابراهيم ،الدليمي ،حنان حامد علي،(2008) ،"استخدام الثبكة العصبية

الاصطناعية نيوكوكنترون في تمييز الرقام العربية المكتوبة يدويا"،المؤتمر العلمي الاول لتقانة

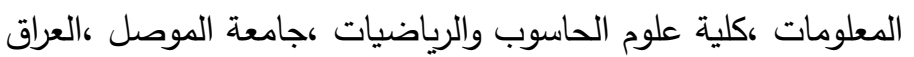

[7] Sushmita Mitrak,Sankar K.Pal and Malay K.kundu,"Fingerprint classification using a fuzy Multilayer perceptron unit,Machine Intelligence unit, Indian statistical Institute, Calcutta,India.

[8] Mehran yazdi,and kazem gheysari, (2008),"A New Approach for the fingerprint classification Based on gray level Co-occurrence Matrix",International Journal of computer and Information Enginerring .

[9] Fakheri,M.M.;Mashoufi,B.Sedghi,T., , (2010),"A Novel Approach for Fingerprinting Recognition",internatonal review on computer \&software.

[10] Adnan Amin , (2004),"fingerprint classification:a revw springer-verlag London, Neil Yager.

[11] Kyuheon Kim, Seyoon Jeong, Byung Tae Chun, Jae Yeon Lee, Younglae Bae, (1999), "Efficient video images retrieval by using local cooccurrence matrix texture features and normalized correlation" ,Proceedings of the IEEE Region 10 Conference TENCON 99. Vol. 2, pp 934-937.

[12] MATLAB help, R2011b,(2011) "neural network toolbox".

[13] Shashi Kumar D R, K B Raja, R K Chhotaray, Sabyasachi Pattanaik, 
(2011), "DWT Based Fingerprint Recognition using Non Minutiae Features ", IJCSI International Journal of Computer Science Issues, Vol. 8, Issue 2.

[14] Sumit Goyal, Gyandera Kumar Goyal, (2011),"Cascade and Feedforward Backpropagation Artificial Neural Network Models For Prediction of Sensory Quality of Instant Coffee Flavoured Sterilized Drink", Canadian Journal on Artificial Intelligence, Machine Learning and Pattern Recognition.

[15] Mark Hudson Beale, Mar tin T. Hag an, Howard B. Demuth, (2012), "Neural Network Toolbox ${ }^{\mathrm{TM}}$ User's Guide R2012b".

[16] Kang Li, Stephen Thompson, (2000), "A Cascaded Neural Network and Its Application to Modelling Power Plant Pollutant Emission", IEEE, Proceedings of the $3 \mathrm{~d}$ World Congress on Intelligent Control and Automation.

[17] H.Ritter, T.Kohonen " Self-Organizing Semantic Maps", Biological Cybernetics, Springer, 241-254.

[18] Al-irhim, Yousra F., (2007),"Corpus Based Arabic Speech Recognition", A Ph.D. thesis, college of computer and mathematical sciences, computer science department, Mosul university.

[19] Al-Gurairi, Maha.A.H,(2006)," Biometric Identification Based on Improved Iris Recognition Techniques", A Ph.D. thesis, college of computer and mathematical sciences, computer science department, Mosul university. 